\title{
Supermesityl-stabilisierte Iminoborane
}

\author{
W. Luthin, G. Elter, A. Heine, D. Stalke, G. M. Sheldrick und A. Meller \\ Göttingen, Institut für Anorganische Chemie der Universität \\ Bei der Redaktion eingegangen am 26. Juni 1991.
}

Inhaltsuibersicht. Amino-imino-borane

$\mathrm{R}^{\prime}\left(\mathrm{SiMe}_{3}\right) \mathrm{N}-\mathrm{B} \equiv \mathrm{N}-\mathrm{R}: \quad$ II $\quad\left(\mathrm{R}^{\prime}=\mathrm{CHMe}_{2}\right)$, II d $\left(\mathrm{R}^{\prime}=\mathrm{CMe}_{3}\right)$ und II e $\left(\mathrm{R}^{\prime}=\mathrm{SiMe}_{3}\right)$ mit $\mathrm{R}=2,4,6-\mathrm{Tr} \mathrm{i}-\mathrm{t}-$ butylphenyl- wurden aus den entsprechenden Fluorbis(amino)boranen Ic-e durch HF-Eliminierung mit tButyllithium (II c, II d) bzw. Lithiumbis(trimethylsilyl)amid (II e) erhalten. II $\mathbf{c}-\mathbf{e}$ sind thermisch stabil und bei Raumtemperatur unbegrenzt haltbar. Die Umsetzun-

\section{Supermesityl stabilized Iminoboranes}

\begin{abstract}
Amino-iminoboranes $\mathrm{R}^{\prime}\left(\mathrm{SiMe}_{3}\right) \mathrm{N}-\mathrm{B} \equiv \mathrm{N}-\mathrm{R}$ : IIc $\left(\mathbf{R}^{\prime}=\mathrm{CHMe}_{2}\right)$, IId $\left(\mathrm{R}^{\prime}=\mathrm{CMe}_{3}\right)$ and II e $\left(\mathrm{R}^{\prime}=\mathrm{SiMe}_{3}\right)$ carrying the supermesityl group $(\mathrm{R})$ on the imino nitrogen atoms have been prepared from the corresponding fluorobis(amino)boranes Ic-e by HF-elimination using t-BuLi (IIc, d) or N-lithio-bis(trimethylsilyl)amid (II e). The Amino-iminoboranes are thermally stable at room temperature. Upon treatment of the fluorobis(amino)boranes $\mathbf{I a}, \mathbf{I b}, \mathbf{I e}$ with $\mathrm{t}-\mathrm{BuLi}, \mathrm{LiF}$ and
\end{abstract}

gen der Fluorbis(amino)borane Ia $\left(\mathrm{R}^{\prime}=\mathrm{Me}\right)$, Ib $\left(\mathrm{R}^{\prime}=\mathrm{CH}_{2} \mathrm{Me}\right)$ und Ie $\left(\mathrm{R}^{\prime}=\mathrm{SiMe}_{3}\right)$ mit $\mathrm{t}$-Butyllithium führen zur Abspaltung von $\mathrm{LiF}$ und $\mathrm{HN}\left(\mathrm{SiMe}_{3}\right) \mathrm{R}^{\prime}$, wobei sich in allen Fällen das B-t-butyl-substituierte Iminoboran III bildet. Die Verbindungen sind elementaranalytisch und spektroskopisch (MS, IR, NMR) charakterisiert. Eine Röntgenstrukturanalyse wurde von IId durchgeführt.

$\mathrm{HN}\left(\mathrm{SiMe}_{3}\right) \mathrm{R}^{\prime}$ are eliminated and the B-t-butyl substituted iminoborane III is formed. The compounds are characterized by elementar analyses and spectroscopic data (MS, IR, NMR). An X-ray diffraction study has been performed for IId.

Key words: 2,4,6-Tri-t-butylphenyl-substituted aminoimino boranes

\section{Einleitung}

Amino-imino-borane, über die unlängst zusammenfassend berichtet wurde $[1,2]$, sind thermodynamisch instabil und neigen bei Raumtemperatur zur Dimerisierung. Durch Einführung sperriger Substituenten sowohl am Amino- als auch am Imino-Stickstoff läßt sich die monomere Species $R_{2}^{\prime} N-B \equiv N-R$ kinetisch stabilisieren, so daß im Falle von $R_{2}^{\prime} N=2,2,6,6$-Tetramethylpiperidinound $R=2,4,6$-Tri-t-butylphenyl- ein Amino-imino-boran erhalten wird, das bei Raumtemperatur monatelang lagerfähig ist und keine Tendenz zur Dimerisierung zeigt [3]. Der dabei an den Iminostickstoff gebundene Supermesitylrest hat sich bei unseren Untersuchungen auch als Substituent am Bor zur Stabilisierung von Iminoboranen bestens bewährt [4-6]. Hier berichten wir über die Herstellung und Struktur weiterer Supermesityl-substituierter Amino-imino-borane sowie die Substitution von sekundären Trimethylsilylaminogruppen durch den $\mathrm{t}$-Butylrest, der bei weniger sterisch anspruchsvollen Zweitsubstituenten am Trimethylsilylamin bei der Umsetzung mit t-Butyllithium erfolgt.

\section{Ergebnisse und Diskussion}

Bis(amino)borane, die einen 2,4,6-Tris(t-butyl)phenylrest an einem Stickstoffatom tragen, können in glatter Reaktion durch Umsetzung von N-Trimethylsilylamino-di- 
fluorboranen mit N-Lithio-2,4,6-tri-t-butylphenylamid erhalten werden (Gl. (1)).

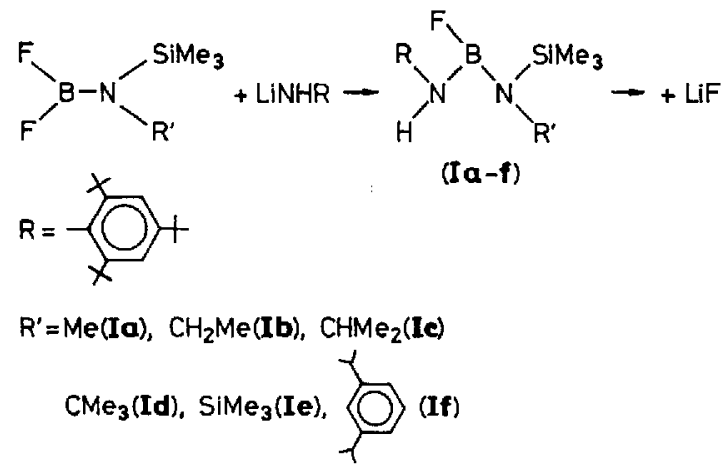

Eine Substitution des zweiten Fluoratoms wird dabei nicht beobachtet; sie ist auch aus sterischen Gründen unwahrscheinlich. Die Verbindungen Ia-f lassen sich im Vakuum unzersetzt als viskose, farblose Flüssigkeiten destillieren, die beim Abkühlen in der Vorlage zu Festkörpern erstarren.

Durch baseninduzierte HF-Eliminierung aus I $\mathbf{c}$ und Id mit Hilfe von t-Butyllithium lassen sich die thermisch sehr stabilen Amino-imino-borane II c, II d in hohen Ausbeuten darstellen (Gl. (2)).

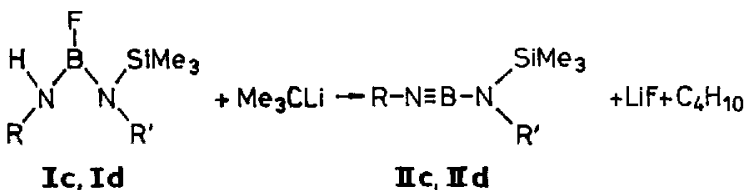

$\mathrm{R}=-\underset{\chi}{\mathrm{O}}+\mathrm{R}^{\prime}=\mathrm{CHMe}_{2}$ (IIc), $\mathrm{CMe}_{3}(\mathrm{IId})$

Dagegen erfolgt bei den Umsetzungen von Ia, Ib und Ie mit $\mathrm{t}$-Butyllithium unter B-N-Spaltung die Eliminierung von $\mathrm{HN}\left(\mathrm{SiMe}_{3}\right) \mathrm{R}^{\prime}$ unter gleichzeitiger Bindung des t-Butylrestes an das Bor und Bildung des Iminoborans III, das schon bei früheren Untersuchungen von uns isoliert wurde $[4,5]$ (Gl. (3)).

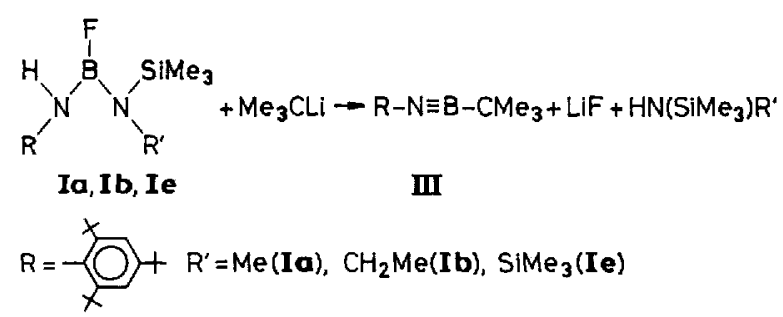

Ein ähnliches Reaktionsverhalten wurde schon bei Umsetzungen von anderen Amino-imino-boranen mit t-Butyllithium beobachtet [7].

Hingegen ergab sich bei der HF-Eliminierung aus Ie mit Lithium-bis(trimethylsilyl)amid das Amino-imino-boran IIe, dessen thermisches Verhalten mit dem von II c und IId vergleichbar ist (Gl. (4)).
Die Amino-imino-borane II c-e lassen sich im Vakuum unzersetzt destillieren. Sie erstarren in der Vorlage, zeigen aber keine Tendenz zur Dimerisierung.

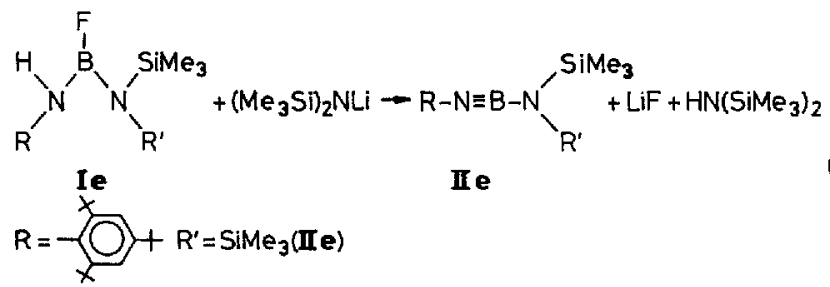

Bei If konnte eine HF-Eliminierung weder mit Methylnoch mit t-Butyllithium erzielt werden. Offensichtlich verhindern die extrem sterisch anspruchsvollen Substituenten einen Angriff der Basen gemäß Gl. (2).

\section{Spektroskopische Charakterisierung}

Die Eliminierung von $\mathrm{HF}$ aus den Bis(amino)boranen Ic-e führt in den ${ }^{11} \mathrm{~B}-\mathrm{NMR}-\mathrm{Spektren}$ der resultierenden Amino-imino-borane zu einer Hochfeldverschiebung um ca. $11 \mathrm{ppm}$. Die so erhaltenen Werte von $+11,8 \mathrm{ppm}$ (II c), 11,9 ppm (II d) und 12,2 ppm (II e) lassen sich ohne Schwierigkeiten in das bislang bekannte Datenmaterial ähnlicher Verbindungen einordnen $[1,7]$.

Dabei läßt sich auch hier beobachten, daß die Lage der ${ }^{11} \mathrm{~B}$-Verschiebungen im wesentlichen von dem Iminosubstituenten bestimmt wird; unterschiedliche Aminoliganden üben dagegen nur geringen Einfluß aus.

Weitgehende Übereinstimmung mit dem Gang der ${ }^{11} \mathrm{~B}$-Resonanzen zeigen auch die IR-Spektren von IIc-e. Die ${ }^{11} \mathrm{~B} \equiv \mathrm{N}$-Valenzschwingung liegt für alle drei Verbindungen - nahezu unbeeinflußt von den unterschiedlichen Aminosubstituenten - lagekonstant um $1990 \mathrm{~cm}^{-1}$. Die entsprechende ${ }^{10} \mathrm{~B} \equiv \mathrm{N}$-Schulter findet sich jeweils $60 \mathrm{~cm}^{-1}$ hochfrequent.

\section{Kristallstruktur von II d ${ }^{1}$ )}

Die Molekülstruktur von II d ist in Abb. 1 wiedergegeben. Die Atomkoordinaten und isotrope Thermalparameter, Bindungsabstände und -winkel sind in den Tab. 1-3 zusammengestellt. Im Vergleich zu den wenigen bekannten Strukturen $[5,8,9]$ von Iminoboranen erhält man bei der hier dargestellten Verbindung entlang der N2B1N1C1-Kette eine innerhalb der Fehlergrenzen lineare NBN-Einheit mit $179,7(5)^{\circ}$, während in den Strukturen von $\mathrm{Me}_{3} \mathrm{C}-\mathrm{N} \equiv \mathrm{B}-\mathrm{Si}\left(\mathrm{SiMe}_{3}\right)_{3}$ [9] bzw. von

1) Weitere Einzelheiten zur Kristallstrukturuntersuchung können beim Fachinformationszentrum Karlsruhe, Gesellschaft für wissenschaftliche Information $\mathrm{mbH}, \mathrm{D}-7514$ Eggenstein-Leopoldshafen 2, unter Angabe der Hinterlegungsnummer CSD-59478, der Autoren und des Zeitschriftenzitats angefordert werden. 


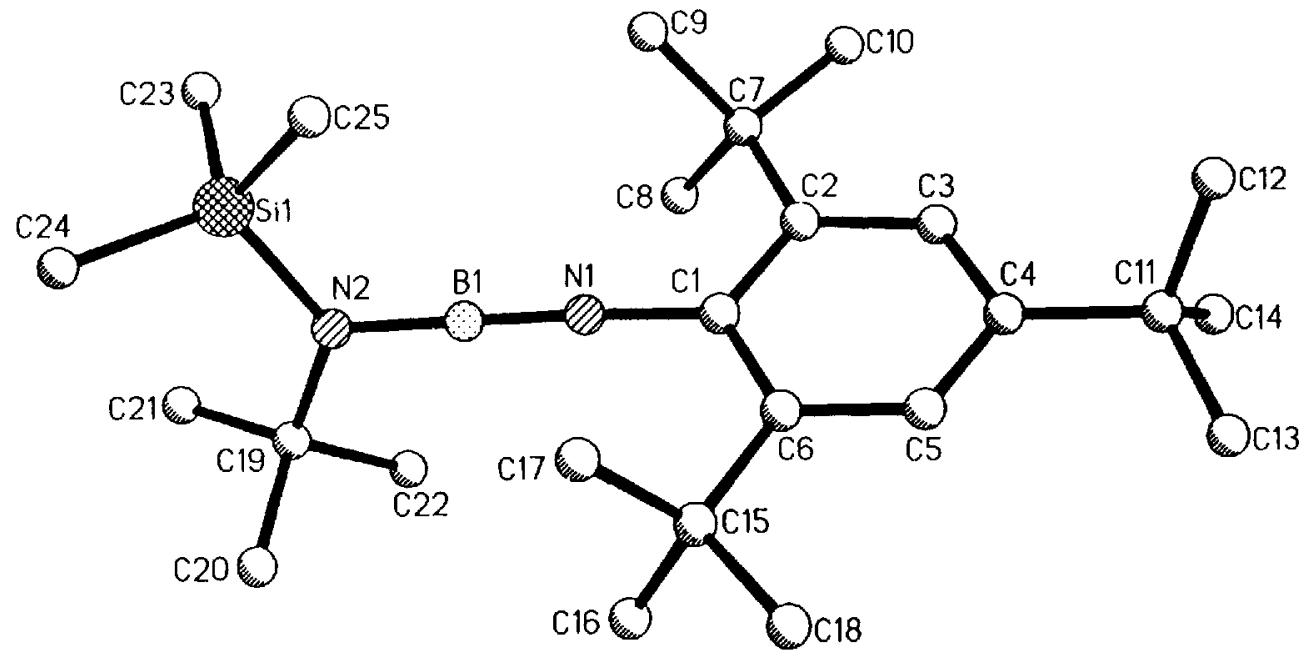

Abb. 1 Molekülstruktur von II d

$\mathrm{Me}_{3} \mathrm{C}-\mathrm{N} \equiv \mathrm{B}-\mathrm{C}_{6} \mathrm{H}_{2}-2,4,6\left(\mathrm{CMe}_{3}\right)_{3} \quad$ [5] NBN-Winkel von ca. $176^{\circ}$ vorliegen. Der $\mathrm{B} 1-\mathrm{N} 1$ Abstand mit 125,3 pm kennzeichnet - zusammen mit dem Bindungswinkel - den Dreifachbindungscharakter des Iminoborans. Auch der B1-N2 Abstand mit 139,2 pm ist gegenüber der BN-Bindungslänge in Monoaminoboranen leicht verkürzt.

Tabelle 1 Atomkoordinaten $\left(\times 10^{4}\right)$ und äquivalente isotrope Auslenkungsparameter $\left(\mathrm{pm}^{2} \times 10^{-1}\right)$ von IId

\begin{tabular}{lrrrr}
\hline & \multicolumn{1}{l}{$\mathrm{x}$} & $\mathrm{y}$ & $\mathrm{z}$ & $\mathrm{U}(\mathrm{eq})$ \\
\hline $\mathrm{Si}(1)$ & $6236(1)$ & $548(1)$ & $1833(1)$ & $34(1)$ \\
$\mathrm{N}(1)$ & $4307(3)$ & $3029(2)$ & $1226(1)$ & $32(1)$ \\
$\mathrm{N}(2)$ & $5763(3)$ & $1151(2)$ & $1190(1)$ & $37(1)$ \\
$\mathrm{B}(1)$ & $5000(5)$ & $2141(4)$ & $1209(2)$ & $33(1)$ \\
$\mathrm{C}(1)$ & $3577(4)$ & $4026(3)$ & $1221(1)$ & $28(1)$ \\
$\mathrm{C}(2)$ & $2191(4)$ & $4006(3)$ & $1365(1)$ & $29(1)$ \\
$\mathrm{C}(3)$ & $1488(4)$ & $5028(3)$ & $1343(2)$ & $32(1)$ \\
$\mathrm{C}(4)$ & $2057(4)$ & $6059(3)$ & $1188(2)$ & $30(1)$ \\
$\mathrm{C}(5)$ & $3400(4)$ & $6053(3)$ & $1050(1)$ & $29(1)$ \\
$\mathrm{C}(6)$ & $4191(3)$ & $5073(3)$ & $1067(1)$ & $28(1)$ \\
$\mathrm{C}(7)$ & $1471(4)$ & $2891(3)$ & $1537(2)$ & $37(1)$ \\
$\mathrm{C}(8)$ & $1493(4)$ & $2022(3)$ & $1048(2)$ & $48(1)$ \\
$\mathrm{C}(9)$ & $2088(4)$ & $2375(4)$ & $2077(2)$ & $48(1)$ \\
$\mathrm{C}(10)$ & $-22(4)$ & $3112(4)$ & $1678(2)$ & $52(2)$ \\
$\mathrm{C}(11)$ & $1219(4)$ & $7161(3)$ & $1181(2)$ & $36(1)$ \\
$\mathrm{C}(12)$ & $844(6)$ & $7463(5)$ & $1786(2)$ & $91(2)$ \\
$\mathrm{C}(13)$ & $1969(5)$ & $8168(4)$ & $927(3)$ & $69(2)$ \\
$\mathrm{C}(14)$ & $-44(4)$ & $6997(4)$ & $829(2)$ & $62(2)$ \\
$\mathrm{C}(15)$ & $5677(4)$ & $5164(3)$ & $910(2)$ & $37(1)$ \\
$\mathrm{C}(16)$ & $5932(4)$ & $4495(4)$ & $353(2)$ & $51(1)$ \\
$\mathrm{C}(17)$ & $6579(4)$ & $4731(4)$ & $1392(2)$ & $54(2)$ \\
$\mathrm{C}(18)$ & $6101(4)$ & $6389(3)$ & $788(2)$ & $50(2)$ \\
$\mathrm{C}(19)$ & $6173(3)$ & $704(2)$ & $612(1)$ & $42(1)$ \\
$\mathrm{C}(20)$ & $7438(5)$ & $1297(5)$ & $382(2)$ & $66(2)$ \\
$\mathrm{C}(21)$ & $6361(6)$ & $-625(4)$ & $607(2)$ & $68(2)$ \\
$\mathrm{C}(22)$ & $4958(5)$ & $950(4)$ & $173(2)$ & $50(1)$ \\
$\mathrm{C}(23)$ & $5196(5)$ & $-655(4)$ & $2019(2)$ & $59(2)$ \\
$\mathrm{C}(24)$ & $7974(4)$ & $129(4)$ & $1850(2)$ & $61(2)$ \\
$\mathrm{C}(25)$ & $5974(5)$ & $1648(4)$ & $2373(2)$ & $55(2)$ \\
\hline
\end{tabular}

äquivalente isotrope $U$ berechnet als ein Drittel der Spur des orthogonalen $\mathbf{U}_{\mathrm{ij}}$ Tensors
Tabelle 2 Bindungsabstände (pm) von II d

\begin{tabular}{llll}
\hline $\mathrm{N}(2)-\mathrm{B}(1)$ & $139,2(5)$ & $\mathrm{N}(1)-\mathrm{B}(1)$ & $125,3(5)$ \\
$\mathrm{N}(1)-\mathrm{C}(1)$ & $137,9(4)$ & $\mathrm{C}(1)-\mathrm{C}(6)$ & $142,0(5)$ \\
$\mathrm{C}(1)-\mathrm{C}(2)$ & $142,9(5)$ & $\mathrm{C}(2)-\mathrm{C}(3)$ & $139,2(5)$ \\
$\mathrm{C}(3)-\mathrm{C}(4)$ & $138,5(5)$ & $\mathrm{C}(4)-\mathrm{C}(5)$ & $138,4(5)$ \\
$\mathrm{C}(5)-\mathrm{C}(6)$ & $139,7(5)$ & $\mathrm{C}(2)-\mathrm{C}(7)$ & $154,8(5)$ \\
$\mathrm{C}(7)-\mathrm{C}(10)$ & $155,4(6)$ & $\mathrm{C}(7)-\mathrm{C}(9)$ & $153,2(6)$ \\
$\mathrm{C}(7)-\mathrm{C}(8)$ & $153,5(6)$ & $\mathrm{C}(4)-\mathrm{C}(11)$ & $154,2(5)$ \\
$\mathrm{C}(11)-\mathrm{C}(14)$ & $152,2(6)$ & $\mathrm{C}(11)-\mathrm{C}(13)$ & $152,0(6)$ \\
$\mathrm{C}(11)-\mathrm{C}(12)$ & $150,8(6)$ & $\mathrm{C}(6)-\mathrm{C}(15)$ & $153,8(5)$ \\
$\mathrm{C}(15)-\mathrm{C}(18)$ & $152,6(6)$ & $\mathrm{C}(15)-\mathrm{C}(17)$ & $153,4(6)$ \\
$\mathrm{C}(15)-\mathrm{C}(16)$ & $154,4(6)$ & & \\
\hline
\end{tabular}

Kristallstrukturdaten. II d: $\mathrm{C}_{25} \mathrm{H}_{47} \mathrm{BN}_{2} \mathrm{Si}, \quad \mathrm{M}=414,5$, ortho-

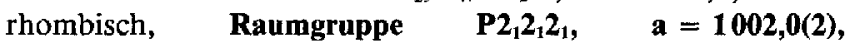
$b=1173,0(2), \quad c=2343,0(5) \mathrm{pm}, \quad V=2,754 \mathrm{~nm}^{3}, \quad Z=4$, $\zeta_{\text {ber. }}=1,00 \mathrm{~g} \mathrm{~cm}^{-3}, \quad \mathrm{~F}(000)=920, \quad \lambda(\mathrm{MoK} \alpha)=71,073 \mathrm{pm}$, $\mu=0,094 \mathrm{~mm}^{-1}$. Kristallgröße $0,6 \times 0,6 \times 0,5 \mathrm{~mm}^{3}$, Meßtemperatur $-120^{\circ} \mathrm{C}$. Stoe-Siemens AED Diffraktometer, 2992 Reflexe mit $2 \Theta=8-50^{\circ}$ gemessen, davon 2733 unabhängige und 2247 beobachtete mit $\mathrm{F}>3 \sigma(\mathrm{F}), \mathrm{R}=0,055, \quad \mathrm{R}_{\mathrm{w}}=0,054$, $\mathrm{w}^{-1}=\sigma^{2}(\mathrm{~F})+0,0007 \mathrm{~F}^{2}$.

Strukturbestimmung. Die Struktur wurde mit Direkten Methoden gelöst und nach dem Kleinste-Quadrate-Verfahren verfeinert. Alle Nicht-Wasserstoffatome wurden anisotrop verfeinert, für die Wasserstoffatome wurde ein Reitermodell verwendet. Die Auslenkungsparameter der Wasserstoffatome wurden isotrop verfeinert, wobei äquivalente Wasserstoffatome auf gleiche Weise festgehalten wurden. Es wurden 263 Parameter verfeinert.

\section{Experimenteller Teil}

Analysen und spektroskopische Messungen: C,H-Bestimmungen wurden im analytischen Labor des Institutes für Anorganische Chemie ausgeführt. Massenspektren: Finnigan MAT 8230. IR-Spektren: Perkin-Elmer 325. NMR-Spektren: Bruker AM 250, Standards: ${ }^{1} \mathrm{H},{ }^{13} \mathrm{C},{ }^{29} \mathrm{Si}$ int. TMS (Kennzeichnung der ${ }^{1} \mathrm{H}$ - und ${ }^{13} \mathrm{C}$ Position erfolgt gemäß den Nummern im folgen- 
Tabelle 3 Bindungswinkel $\left({ }^{\circ}\right)$ von II d

\begin{tabular}{llll}
\hline$N(1)-B(1)-N(2)$ & $179,7(5)$ & $\mathrm{Si}(1)-N(2)-C(19)$ & $124,5(2)$ \\
$\mathrm{Si}(1)-\mathrm{N}(2)-\mathrm{B}(1)$ & $117,6(3)$ & $\mathrm{C}(19)-\mathrm{N}(2)-\mathrm{B}(1)$ & $117,8(3)$ \\
$\mathrm{C}(1)-\mathrm{N}(1)-\mathrm{B}(1)$ & $177,3(4)$ & $\mathrm{N}(1)-\mathrm{C}(1)-\mathrm{C}(6)$ & $120,3(3)$ \\
$\mathrm{N}(1)-\mathrm{C}(1)-\mathrm{C}(2)$ & $120,0(3)$ & $\mathrm{C}(6)-\mathrm{C}(1)-\mathrm{C}(2)$ & $119,7(3)$ \\
$\mathrm{C}(1)-\mathrm{C}(6)-\mathrm{C}(5)$ & $118,2(3)$ & $\mathrm{C}(1)-\mathrm{C}(6)-\mathrm{C}(15)$ & $122,7(3)$ \\
$\mathrm{C}(5)-\mathrm{C}(6)-\mathrm{C}(15)$ & $119,0(3)$ & $\mathrm{C}(6)-\mathrm{C}(5)-\mathrm{C}(4)$ & $123,3(3)$ \\
$\mathrm{C}(1)-\mathrm{C}(2)-\mathrm{C}(3)$ & $118,0(3)$ & $\mathrm{C}(1)-\mathrm{C}(2)-\mathrm{C}(7)$ & $121,9(3)$ \\
$\mathrm{C}(3)-\mathrm{C}(2)-\mathrm{C}(7)$ & $120,1(3)$ & $\mathrm{C}(2)-\mathrm{C}(3)-\mathrm{C}(4)$ & $123,6(3)$ \\
$\mathrm{C}(5)-\mathrm{C}(4)-\mathrm{C}(3)$ & $117,1(3)$ & $\mathrm{C}(5)-\mathrm{C}(4)-\mathrm{C}(11)$ & $122,1(3)$ \\
$\mathrm{C}(3)-\mathrm{C}(4)-\mathrm{C}(11)$ & $120,7(3)$ & $\mathrm{C}(2)-\mathrm{C}(7)-\mathrm{C}(10)$ & $111,3(3)$ \\
$\mathrm{C}(2)-\mathrm{C}(7)-\mathrm{C}(9)$ & $111,2(3)$ & $\mathrm{C}(10)-\mathrm{C}(7)-\mathrm{C}(9)$ & $106,2(3)$ \\
$\mathrm{C}(2)-\mathrm{C}(7)-\mathrm{C}(8)$ & $111,1(3)$ & $\mathrm{C}(10)-\mathrm{C}(7)-\mathrm{C}(8)$ & $106,4(3)$ \\
$\mathrm{C}(9)-\mathrm{C}(7)-\mathrm{C}(8)$ & $110,4(3)$ & $\mathrm{C}(4)-\mathrm{C}(11)-\mathrm{C}(14)$ & $110,6(3)$ \\
$\mathrm{C}(4)-\mathrm{C}(11)-\mathrm{C}(13)$ & $112,7(3)$ & $\mathrm{C}(14)-\mathrm{C}(11)-\mathrm{C}(13)$ & $107,4(4)$ \\
$\mathrm{C}(4)-\mathrm{C}(11)-\mathrm{C}(12)$ & $108,8(3)$ & $\mathrm{C}(14)-\mathrm{C}(11)-\mathrm{C}(12)$ & $109,3(4)$ \\
$\mathrm{C}(13)-\mathrm{C}(11)-\mathrm{C}(12)$ & $107,9(4)$ & $\mathrm{C}(6)-\mathrm{C}(15)-\mathrm{C}(18)$ & $112,3(3)$ \\
$\mathrm{C}(6)-\mathrm{C}(15)-\mathrm{C}(17)$ & $111,8(3)$ & $\mathrm{C}(18)-\mathrm{C}(15)-\mathrm{C}(17)$ & $106,6(3)$ \\
$\mathrm{C}(6)-\mathrm{C}(15)-\mathrm{C}(16)$ & $109,1(3)$ & $\mathrm{C}(18)-\mathrm{C}(15)-\mathrm{C}(16)$ & $105,9(3)$ \\
$\mathrm{C}(17)-\mathrm{C}(15)-\mathrm{C}(16)$ & $110,9(3)$ & & \\
\hline
\end{tabular}

den Formelbild $+{ }_{5}^{3}$

$\mathrm{C}_{6} \mathrm{~F}_{6} ;{ }^{15} \mathrm{~N}$ ext. $\mathrm{MeNO}_{2}$. Alle Versuche wurden in einer Inertgasatmosphäre $\left(\mathrm{N}_{2}\right)$ durchgeführt.

Ausgangsverbindungen: $\mathrm{F}_{2} \mathrm{BNSiMe}_{3} \mathrm{R}^{\prime}\left(\mathrm{R}^{\prime}=\mathrm{Me}\right.$ [10], $\mathrm{CH}_{2} \mathrm{Me}$ [10], $\mathrm{CHMe}_{2}$ [10], $\mathrm{SiMe}_{3}$ [10], $\mathrm{C}_{6} \mathrm{H}_{3}\left(\mathrm{CHMe}_{2}\right)_{2}$ [11]. 2,4,6-Tri(t-butylphenyl)lithiumamid wurde durch Reaktion von 2,4,6Tri-t-butylanilin mit Methyllithium in Toluol erhalten.

Darstellung der Verbindungen Ia-f nach $G l$. (l): Zu einer Aufschlämmung von $0,03 \mathrm{~mol} 2,4,6$-Tri(t-butylphenyl)lithiumamid in $100 \mathrm{ml}$ Toluol tropft man bei $-50^{\circ} \mathrm{C} 0,03 \mathrm{~mol}$ Alkyl(trimethylsilyl)aminodifluorboran, gelöst in $50 \mathrm{ml}$ Toluol. Nach dem Auftauen wird noch ca. $3 \mathrm{~h}$ bei Raumtemperatur gerührt. Das Lösungmittel wird im Vakuum abgezogen und der verbleibende Rückstand bei 0,01 mbar destilliert.

(2,4,6-Tri-t-butylphenylamino)-fluor-[methy](trimethylsilyl)aminolboran (Ia):

Ausbeute $90 \%$; Sdp. $100{ }^{\circ} \mathrm{C} / 0,01$ mbar.

Analyse: $\mathrm{C}_{22} \mathrm{H}_{42} \mathrm{BN}_{2} \mathrm{FSi}(392,46)$; $\mathrm{C} 67,26$ (ber. 67,33$), \mathrm{H} 10,62$ (ber. 10,79).

MS: $392 \mathrm{M}^{+}(48 \%), 377 \mathrm{M}-\mathrm{Me}^{+}(30 \%), 57 \mathrm{CMe}_{3}{ }^{+}(100 \%)$.

'H-NMR: $\delta=7,35$ (s, 2H, 3/5), 3,74 (d, ${ }^{3} J_{\mathrm{HF}}=17,3 \mathrm{~Hz}, 1 \mathrm{H}$, $\mathrm{NH}), 2,68$ (s, 3H, NMe), 1,43 (s, 18H, $\mathrm{CMe}_{3}$ an 2/6), 1,31 (s, 9H, $\mathrm{CMe}_{3}$ an 4), $0,14\left(\mathrm{~d},{ }^{5} \mathrm{~J}_{\mathrm{HF}}=1,5 \mathrm{~Hz}, 9 \mathrm{H}, \mathrm{SiMe}_{3}\right)$.

${ }^{13} \mathrm{C}-\mathrm{NMR}: \delta=147,8(2 / 6), 146,8(4), 136,3(1), 122,3(3 / 5), 36,2$ $\left(\mathrm{CMe}_{3}\right.$ an 2/6), 34,9 (CMe $\mathrm{CM}_{3}$ ), 31,9 $\left(\mathrm{CMe}_{3}\right.$ an $\left.2 / 6\right), 31,6$ $\left(\mathrm{CMe}_{3}\right.$ an 4), 29,9 (d, $\left.{ }^{3} J_{C F}=6,1 \mathrm{~Hz}, \mathrm{NMe}\right), \quad 0,4 \quad(d$, ${ }^{4} \mathbf{J}_{\mathrm{CF}}=2,9 \mathrm{~Hz}, \mathrm{SiMe}_{3}$ ).

${ }^{11} \mathrm{~B}-\mathrm{NMR}: \delta=+23,5$.

${ }^{19} \mathrm{~F}-\mathrm{NMR}: \delta=37,3\left(\mathrm{~d},{ }^{3} \mathrm{~J}_{\mathrm{HF}}=17,3 \mathrm{~Hz}\right)$.

${ }^{29} \mathrm{Si}-\mathrm{NMR}: \delta=+9,9\left(\mathrm{~d},{ }^{3} \mathrm{~J}_{\mathrm{SiF}}=3,5 \mathrm{~Hz}\right)$.

(2,4,6-Tri-t-butylphenylamino)-[ethyl(trimethylsilyl)amino] fluorboran (I b)

Ausbeute $90 \%$; Sdp. $129^{\circ} \mathrm{C} / 0,01$ mbar.
Analyse: $\mathrm{C}_{23} \mathrm{H}_{44} \mathrm{BN}_{2} \mathrm{FSi}(406,52) ; \mathrm{C} 66,86$ (ber. 67,96), $\mathrm{H} 10,80$ (ber. 10,91).

MS: $406 \mathrm{M}^{+}(18 \%), 391 \mathrm{M}-\mathrm{Me}^{+}(12 \%), 57 \mathrm{CMe}_{3}{ }^{+}(100 \%)$. 'H-NMR: $\delta=7,35(\mathrm{~s}, 2 \mathrm{H}, 3 / 5), 3,71\left(\mathrm{~d},{ }^{3} \mathrm{~J}_{\mathrm{HF}}=17,6 \mathrm{~Hz}, 1 \mathrm{H}\right.$, $\mathrm{NH}), 3,04\left(\mathrm{q},{ }^{3} \mathrm{~J}_{\mathrm{HH}}=7,1 \mathrm{~Hz}, 2 \mathrm{H}, \mathrm{CH}_{2} \mathrm{Me}\right.$ ), 1,44 (s, $18 \mathrm{H}, \mathrm{CMe}_{3}$ an $2 / 6), 1,31\left(\mathrm{~s}, 9 \mathrm{H}, \mathrm{CMe}_{3}\right.$ an 4$), 1,20\left(\mathrm{t},{ }^{3} \mathrm{~J}_{\mathrm{HH}}=7,1 \mathrm{~Hz}, 3 \mathrm{H}\right.$, $\left.\mathrm{CH}_{2} \mathrm{Me}\right), 0,15$ (d, ${ }^{5} \mathrm{~J}_{\mathrm{HF}}=1,4 \mathrm{~Hz}, 9 \mathrm{H}, \mathrm{SiMe}_{3}$ ).

${ }^{13} \mathrm{C}-\mathrm{NMR}: \delta=148,0(2 / 6), 147,0(4), 136,4\left(\mathrm{~d},{ }^{3} \mathrm{~J}_{\mathrm{CF}}=1,2 \mathrm{~Hz}\right.$, 1), $122,4(3 / 5), 38,1\left(\mathrm{~d},{ }^{3} \mathrm{~J}_{\mathrm{CF}}=5,7 \mathrm{~Hz}, \mathrm{CH}_{2} \mathrm{Me}\right), 36,3\left(\mathrm{CMe}_{3}\right.$ an

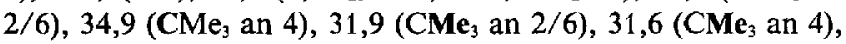
$19,3\left(\mathrm{CH}_{2} \mathrm{Me}\right), 0,9\left(\mathrm{~d},{ }^{4} \mathrm{~J}_{\mathrm{CF}}=2,8 \mathrm{~Hz}, \mathrm{SiMe}_{3}\right)$.

${ }^{11} \mathrm{~B}-\mathrm{NMR}: \delta=+23,6$.

${ }^{19} \mathrm{~F}-\mathrm{NMR}: \delta=+38,3\left(\mathrm{~d},{ }^{3} \mathrm{~J}_{\mathrm{HF}}=17,6 \mathrm{~Hz}\right)$.

${ }^{29} \mathrm{Si}-\mathrm{NMR}: \delta=+9,2\left(\mathrm{~d},{ }^{3} \mathrm{~J}_{\mathrm{SiF}}=4,8 \mathrm{~Hz}\right)$.

(2,4,6-Tri-t-butylphenylamino)-fluor-[isopropyl(trimethylsilyl)aminolboran (I c)

Ausbeute $85 \%$; Sdp. $134^{\circ} \mathrm{C} / 0,01 \mathrm{mbar}$.

Analyse: $\mathrm{C}_{24} \mathrm{H}_{46} \mathrm{BN}_{2} \mathrm{FSi}(420,54)$; $\mathrm{C} 67,82$ (ber. 68,55$), \mathrm{H} 10,99$ (ber. 11,03).

MS: $420 \mathrm{M}^{+}(28 \%), 405 \mathrm{M}-\mathrm{Me}^{+}(8 \%), 57 \mathrm{CMe}_{3}{ }^{+}(100 \%)$.

${ }^{1} \mathrm{H}-\mathrm{NMR}: \delta=7,36(\mathrm{~s}, 2 \mathrm{H}, 3 / 5), 3,74\left(\mathrm{~d},{ }^{3} \mathrm{~J}_{\mathrm{HF}}=18,5 \mathrm{~Hz}, 1 \mathrm{H}\right.$, $\mathrm{NH}$ ), 3,39 (sept, $\left.{ }^{3} \mathrm{~J}_{\mathrm{HH}}=6,9 \mathrm{~Hz}, 1 \mathrm{H}, \mathrm{CHMe}_{2}\right), 1,44(\mathrm{~s}, 18 \mathrm{H}$, $\mathrm{CMe}_{3}$ an 2/6), 1,31 (s, 9H, $\mathrm{CMe}_{3}$ an 4), 1,30 (d, ${ }^{3} \mathrm{~J}_{\mathrm{HH}}=6,9 \mathrm{~Hz}$, 6H, $\left.\mathrm{CHMe}_{2}\right), 0,22\left(\mathrm{br}, 9 \mathrm{H}, \mathrm{SiMe}_{3}\right)$.

${ }^{13} \mathrm{C}-\mathrm{NMR}: \delta=147,8(2 / 6), 146,6(4), 136,4\left(\mathrm{~d},{ }^{3} \mathrm{~J}_{\mathrm{CF}}=1,8 \mathrm{~Hz}\right.$, 1), $122,4(2 / 5), 46,2\left(\mathrm{~d},{ }^{3} \mathrm{~J}_{\mathrm{CF}}=2,8 \mathrm{~Hz}, \mathrm{CHMe}_{2}\right), 36,2\left(\mathrm{CMe}_{3}\right.$ an 2/6), 34,8 ( $\mathrm{CMe}_{3}$ an 4), 31,9 (CMe 3 an 2/6), 31,6 (CMe 3 an 4), $24,9\left(\mathrm{CHMe}_{2}\right), 1,9\left(\mathrm{~d},{ }^{4} \mathrm{~J}_{\mathrm{CF}}=2,3 \mathrm{~Hz}, \mathrm{SiMe}_{3}\right)$.

${ }^{11} \mathrm{~B}-\mathrm{NMR}: \delta=+23,1$.

${ }^{19} \mathrm{~F}-\mathrm{NMR}: \delta=+45,3\left(\mathrm{~d},{ }^{3} \mathrm{~J}_{\mathrm{HF}}=18,5 \mathrm{~Hz}\right)$.

${ }^{29}$ Si-NMR: $\delta=+6,4$ (br).

[t-Butyl(trimethylsilyl)amino](2,4,6-tri-t-butylphenylamino) fluorboran (I d)

Ausbeute $80 \%$, Sdp. $141^{\circ} \mathrm{C} / 0,01$ mbar.

Analyse: $\mathrm{C}_{25} \mathrm{H}_{48} \mathrm{BN}_{2} \mathrm{FSi}(434,57) ; \mathrm{C} 68,12$ (ber. 69,10), $\mathrm{H} \mathrm{10,97}$ (ber, 11,13).

MS: $434 \mathrm{M}^{+}(6 \%), 57 \mathrm{CMe}_{3} \quad(100 \%)$. 
${ }^{1} \mathrm{H}-\mathrm{NMR}: \delta=7,35(\mathrm{~s}, 2 \mathrm{H}, 3 / 5), 4,02\left(\mathrm{~d},{ }^{3} \mathrm{~J}_{\mathrm{HF}}=19,5 \mathrm{~Hz}, 1 \mathrm{H}\right.$, $\mathrm{NH}), 1,45$ (s, 27H, CMe 3 an $\left.2 / 6, \mathrm{NCMe}_{3}\right), 1,31$ (s, 9H, $\mathrm{CMe}_{3}$ an 4), 0,30 (d, $\left.{ }^{5} \mathrm{HF}=1,8 \mathrm{~Hz}, 9 \mathrm{H}, \mathrm{SiMe}_{3}\right)$.

${ }^{13} \mathrm{C}-\mathrm{NMR}: \delta=146,8(2,6), 146,1(4), 136,6\left(\mathrm{~d},{ }^{3} \mathrm{~J}_{\mathrm{CF}}=2,3 \mathrm{~Hz}, 1\right)$, $122,3(3 / 5), 53,3\left(\mathrm{NCMe}_{3}\right), 36,2\left(\mathrm{CMe}_{3}\right.$ an 2/6), 34,7 $\left(\mathrm{CMe}_{3}\right.$ an 4), 33,9 (d, $\left.{ }^{4} J_{\mathrm{CF}}=2,3 \mathrm{~Hz}, \mathrm{NCMe}_{3}\right), 32,1\left(\mathrm{CMe}_{3}\right.$ an $\left.2 / 6\right), 31,6$ $\left(\mathrm{CMe}_{3}\right.$ an 4$), 6,1\left(\mathrm{~d},{ }^{4} \mathrm{~J}_{\mathrm{CF}}=4,6 \mathrm{~Hz}, \mathrm{SiMe}_{3}\right)$.

"B-NMR: $\delta=+23,0$.

${ }^{19}$ F-NMR: $\delta=+56,2\left(\mathrm{~d},{ }^{3} \mathrm{~J}_{\mathrm{HF}}=19,5 \mathrm{~Hz}\right)$.

${ }^{29}$ Si-NMR: $\delta=+4,0$ (br).

${ }^{15} \mathrm{~N}-\mathrm{NMR}: \delta=-302(\mathrm{NH})$.

(2,4,6-Tri-t-butylphenylamino)-fluor-[bis(trimethylsilyl)aminolboran (I e)

Ausbeute $90 \%$, Kp. $139^{\circ} \mathrm{C} / 0,01 \mathrm{mbar}$.

Analyse: $\mathrm{C}_{24} \mathrm{H}_{48} \mathrm{BN}_{2} \mathrm{FSi}_{2}(450,64) ; \mathrm{C} 63,81$ (ber. 63,97), $\mathrm{H} 10,71$ (ber. 10,74).

MS: $450 \mathrm{M}^{+}(98 \%), 435 \mathrm{M}-\mathrm{Me}^{+}(20 \%), 57 \mathrm{CMe}_{3}{ }^{+}(100 \%)$.

${ }^{1} \mathrm{H}-\mathrm{NMR}: \delta=7,35(\mathrm{~s}, 2 \mathrm{H}, 3 / 5), 4,06\left(\mathrm{~d},{ }^{3} \mathrm{~J}_{\mathrm{HF}}=19,2 \mathrm{~Hz}, 1 \mathrm{H}\right.$, $\mathrm{NH}$ ), 1,43 (s, 18H, $\mathrm{CMe}_{3}$ an 2/6), 1,31 (s, 9H, $\mathrm{CMe}_{3}$ an 4 ), $0,26\left(\mathrm{~d},{ }^{5} \mathbf{J}_{\mathrm{HF}}=1,1 \mathrm{~Hz}, 18 \mathrm{H}, \mathrm{SiMe}_{3}\right)$.

${ }^{13} \mathrm{C}-\mathrm{NMR}: \delta=147,3(2 / 6), 146,7(4), 136,2\left(\mathrm{~d},{ }^{3} \mathrm{~J}_{\mathrm{CF}}=1,7 \mathrm{~Hz}\right.$, 1), $122,5(3 / 5), 36,3\left(\mathrm{CMe}_{3}\right.$ an 2/6), 34,8 (CMe ${ }_{3}$ an 4$), 32,2$ (d, ${ }^{6} \mathrm{~J}_{\mathrm{CF}}=0,9 \mathrm{~Hz}, \mathrm{CMe}_{3}$ an 2/6), 31,6 (CMe ${ }_{3}$ an 4$), 4,2(\mathrm{~d}$, $\left.{ }^{4} J_{C F}=2,5 \mathrm{~Hz}, \mathrm{SiMe}_{3}\right)$.

${ }^{11} \mathrm{~B}-\mathrm{NMR}: \delta=+23,3$.

${ }^{19}$ F-NMR: $\delta=+58,8\left(\mathrm{~d},{ }^{3} \mathrm{~J}_{\mathrm{HF}}=19,2 \mathrm{Jz}\right)$.

${ }^{29} \mathrm{Si}-\mathrm{NMR}: \delta=+5,1\left(\mathrm{~d},{ }^{3} \mathrm{~J}_{\mathrm{SiF}}=10,4 \mathrm{~Hz}\right)$.

(2,4,6-Tri-t-butylphenylamino)-fluor-[2,6-diisopropylphenyl(trimethylsilyl)amino]boran (If)

Ausbeute $75 \%$, Kp. $210^{\circ} \mathrm{C} / 0,01 \mathrm{mbar}$.

Analyse: $\mathrm{C}_{33} \mathrm{H}_{56} \mathrm{BN}_{2} \mathrm{FSi}(538,72)$; C 72,42 (ber. 73,58 ), $\mathrm{H} 10,12$ (ber. 10,48).

MS: $538 \mathrm{M}^{+}(34 \%), 523 \mathrm{M}-\mathrm{Me}^{+}(89 \%), 73 \mathrm{Me}_{3} \mathrm{Si}^{+}(100 \%)$.

'H-NMR: $\quad \mathrm{RNH}-\delta=7,35 \quad(\mathrm{~s}, 2 \mathrm{H}, 3 / 5), 3,83 \quad$ (d, $\left.{ }^{3} \mathrm{~J}_{\mathrm{HF}}=17,5 \mathrm{~Hz}, 1 \mathrm{H}, \mathrm{NH}\right), 1,49\left(\mathrm{~s}, 18 \mathrm{H}, \mathrm{CMe}_{3}\right.$ an $\left.2 / 6\right), 1,30(\mathrm{~s}$, $9 \mathrm{H}, \mathrm{CMe}_{3}$ an 4).

${ }^{13} \mathrm{C}-\mathrm{NMR}: \delta=147,5(2 / 6), 146,7(4), 135,7(1), 122,4(3 / 5), 36,4$

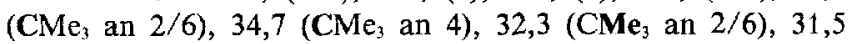
$\left(\mathrm{CMe}_{3}\right.$ an 4$)$.

'H-NMR: RNSiMe ${ }_{3}-\delta=7,1\left(\mathrm{AB}_{2}, 2 \mathrm{H}, 3 / 4 / 5\right), 3,52$ (sept., $\left.{ }^{3} \mathrm{~J}_{\mathrm{HH}}=6,8 \mathrm{~Hz}, 2 \mathrm{H}, C \mathrm{HMe}_{2}\right), 1,23\left(\mathrm{~d},{ }^{3} \mathrm{~J}_{\mathrm{HH}}=6,8 \mathrm{~Hz}, 6 \mathrm{H}\right.$, $\left.\mathrm{CHMe}_{2}\right), 1,16\left(\mathrm{~d},{ }^{3} \mathrm{~J}_{\mathrm{HH}}=6,8 \mathrm{~Hz}, 6 \mathrm{H}, \mathrm{CHMe}_{2}\right), 0,21(\mathrm{~s}, 9 \mathrm{H}$, $\mathrm{SiMe}_{3}$ ).

${ }^{13} \mathrm{C}-\mathrm{NMR}: \quad \delta=146,3(2 / 6), \quad 139,0 \quad\left(\mathrm{~d}, \quad{ }^{3} \mathrm{~J}_{\mathrm{CF}}=4,5 \mathrm{~Hz}, \quad 1\right)$, $125,5(4), \quad 123,7(3 / 5), 27,6 \quad\left(\mathrm{CHMe}_{2}\right), 25,8 \quad\left(\mathrm{CHMe}_{2}\right), 24,7$ $\left(\mathrm{CHMe}_{2}\right), 1,8\left(\mathrm{~d},{ }^{4} \mathrm{~J}_{\mathrm{CF}}=1,2 \mathrm{~Hz}, \mathrm{SiMe}\right)$.

"B-NMR: $\delta=+21,9$.

${ }^{19} \mathrm{~F}-\mathrm{NMR}: \delta=+43,4\left(\mathrm{~d},{ }^{3} \mathrm{~J}_{\mathrm{Hr}}=17,5 \mathrm{~Hz}\right)$.

${ }^{29} \mathrm{Si}-\mathrm{NMR}: \delta=+7,5$ (br).

Darstellung der Verbindungen II $\mathrm{c}-\mathrm{e}$ nach Gl. 2,4: $0,03 \mathrm{Mol}$ Ic-e werden in $150 \mathrm{ml}$ Toluol gelöst und bei RT mit $0,03 \mathrm{~mol}$ Lithiumverbindung versetzt.

Ic: $15 \%$ ige Lösung von t-Butyllithium in Pentan. Anschließend wird $3 \mathrm{~h}$ bei RT gerührt.

Id: $15 \%$ ige Lösung von t-Butyllithium in Pentan. Anschließend wird das Pentan abdestilliert und noch $10 \mathrm{~h}$ unter Rückfluß erhitzt.
Ie: $0,03 \mathrm{Mol}$ Lithium-bis(trimethylsilyl)amid, gelöst in $50 \mathrm{ml}$ Toluol. Anschließend wird $10 \mathrm{~h}$ unter Rückfluß erhitzt.

Das Lösungsmittel wird abgezogen und der verbleibende Rückstand bei 0,01 mbar fraktioniert destilliert. Die Aminoimino-borane II c-e fallen als klare Flüssigkeiten an, die in der Vorlage rasch zu Festkörpern erstarren.

\section{(2,4,6-Tri-t-butylphenylimino)[i-propyl(trimethylsilyl)ami-} nolboran (II c)

Ausbeute $90 \%$, Kp. $129^{\circ} \mathrm{C} / 0,01$ mbar.

Analyse: $\mathrm{C}_{24} \mathrm{H}_{45} \mathrm{BN}_{2} \mathrm{Si}(400,54)$; $\mathrm{C} 71,11$ (ber. 71,97), $\mathrm{H} 11,17$ (ber. 11,32).

MS: $400 \mathrm{M}^{+}(60 \%), 385 \mathrm{M}-\mathrm{Me}^{+}(100 \%)$.

IR: $v\left({ }^{11} \mathrm{~B} \equiv \mathrm{N}\right)=1991 \mathrm{~cm}^{-1}, v\left({ }^{10} \mathrm{~B} \equiv \mathrm{N}\right)=2052 \mathrm{~cm}^{-1}$.

${ }^{1} \mathrm{H}$-NMR: $\delta=7,30$ (s, 2H, 3/5), 3,25 (sept., ${ }^{3} J_{\mathrm{HH}}=6,6 \mathrm{~Hz}$, $\left.1 \mathrm{H}, \mathrm{CHMe}_{2}\right), 1,52\left(\mathrm{~s}, 18 \mathrm{H}, \mathrm{CMe}_{3}\right.$ an $\left.2 / 6\right), 1,31\left(\mathrm{~s}, 9 \mathrm{H}, \mathrm{CMe}_{3}\right.$ an 4), 1,30 (d, $\left.{ }^{3} \mathrm{~J}_{\mathrm{HH}}=6,6 \mathrm{~Hz}, 6 \mathrm{H}, \mathrm{CHMe}_{2}\right), 0,23(\mathrm{~s}, 9 \mathrm{H}$, $\mathrm{SiMe}_{3}$ ).

${ }^{13} \mathrm{C}-\mathrm{NMR}: \delta=144,8(2 / 6), 142,5(4), 135,6(1), 121,3(3 / 5), 47,5$

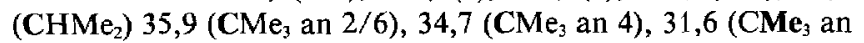

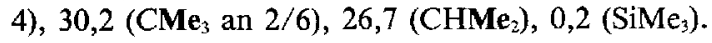

"B-NMR: $\delta=11,8$.

${ }^{15} \mathrm{~N}-\mathrm{NMR}: \delta=-330,8,-275,4$.

${ }^{29} \mathrm{Si}-\mathrm{NMR}: \delta=+10,0$

[t-Butyl(trimethylsilyl)amino](2,4,6-tri-t-butylphenylimino)boran (II d)

Ausbeute $85 \%$, Kp. $141{ }^{\circ} \mathrm{C} / 0,01$ mbar.

Analyse: $\mathrm{C}_{25} \mathrm{H}_{47} \mathrm{BN}_{2} \mathrm{Si}(414,56)$; $\mathrm{C} 71,99$ (ber. 72,43), H 11,19 (ber. 11,43 ).

MS: $414 \mathrm{M}^{+}(30 \%), 399 \mathrm{M}-\mathrm{Me}^{+}(55 \%), 57 \mathrm{CMe}_{3}{ }^{+}(100 \%)$.

IR: $v\left({ }^{11} \mathrm{~B} \equiv \mathrm{N}\right)=1984 \mathrm{~cm}^{-1}, v\left({ }^{10} \mathrm{~B} \equiv \mathrm{N}\right)=2041 \mathrm{~cm}^{-1}$.

${ }^{1} \mathrm{H}$-NMR: $\delta=7,30(\mathrm{~s}, 2 \mathrm{H}, 3 / 5), 1,52\left(\mathrm{~s}, 18 \mathrm{H}, \mathrm{CMe}_{3}\right.$ an $\left.2 / 6\right)$, 1,42 (s, 9H, $\mathrm{NCMe}_{3}$ ), 1,31 (s, 9H, $\mathrm{CMe}_{3}$ an 4), 0,31 (s, 9H, $\left.\mathrm{SiMe}_{3}\right)$.

${ }^{13} \mathrm{C}-\mathrm{NMR}: \delta=145,0(2 / 6), 142,3(4), 135,7(1), 121,4(3 / 5), 55,1$ $\left(\mathrm{NCMe}_{3}\right), 35,9\left(\mathrm{CMe}_{3}\right.$ an $\left.2 / 6\right), 34,7\left(\mathrm{CMe}_{3}\right.$ an 4$), 33,3$

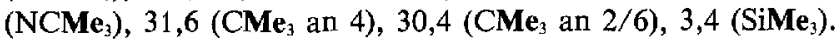

${ }^{: 1} \mathrm{~B}-\mathrm{NMR}: \delta=+11,9$.

${ }^{15} \mathrm{~N}-\mathrm{NMR}: \delta=-321,0,-275,4$.

${ }^{29} \mathrm{Si}-\mathrm{NMR}: \delta=+5,4$.

\section{(2,4,6-Tri-t-butylphenylimino)[bis(trimethylsilyl)amino]boran}

(II e):

Ausbeute $90 \%$, Kp. $143^{\circ} \mathrm{C} / 0,01 \mathrm{mbar}$.

Analyse: $\mathrm{C}_{24} \mathrm{H}_{47} \mathrm{BN}_{2} \mathrm{Si}_{2}(430,64) ; \mathrm{C} 65,77$ (ber. 66,94), $\mathrm{H} 10,52$

(ber. 11,00 ).

MS: $430 \mathrm{M}^{+}(98 \%), 415 \mathrm{M}-\mathrm{Me}^{+}(20 \%), 57 \mathrm{CMe}_{3}{ }^{+}(100 \%)$.

IR: $v\left({ }^{11} \mathrm{~B} \equiv \mathrm{N}\right)=1988 \mathrm{~cm}^{-1}, v\left({ }^{10} \mathrm{~B} \equiv \mathrm{N}\right)=2047 \mathrm{~cm}^{-1}$.

${ }^{1} \mathrm{H}-\mathrm{NMR}: \delta=7,29(\mathrm{~s}, 2 \mathrm{H}, 3 / 5), 1,52\left(\mathrm{~s}, 18 \mathrm{H}, \mathrm{CMe}_{3}\right.$ an $\left.2 / 6\right)$, 1,32 (s, 9H, $\mathrm{CMe}_{3}$ an 4), 0,29 (s, 9H, $\left.\mathrm{SiMe}_{3}\right)$.

${ }^{13} \mathrm{C}-\mathrm{NMR}: \delta=144,5(2 / 6), 142,1(4), 135,6(1), 121,3(3 / 5), 35,9$

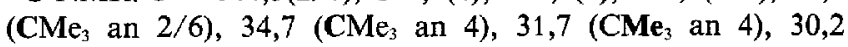
$\left(\mathrm{CMe}_{3}\right.$ an $\left.2 / 6\right)$, ( $\left.\mathrm{SiMe}_{3}\right)$.

${ }^{1} \mathrm{~B}-\mathrm{NMR}: \delta=+12,2$.

${ }^{15} \mathrm{~N}-\mathrm{NMR}: \delta=-348,4,-282,4$.

${ }^{29}$ Si-NMR: $\delta=+10,0$.

Für die Förderung dieser Arbeit danken wir dem Fonds der Chemischen Industrie. 


\section{Literatur}

[1] H. Nöth: Angew. Chem. 100 (1988) 1664

[2] P. Paetzold: Adv. Inorg. Chem. 31 (1987) 123

[3] H. Nöth, B. Rasthofer, S. Weber, Ch. Narula, A. Konstantinov: Pure Appl. Chem. 55 (1983) 1453

[4] M. Neuhaus: Dissertation, Universität Göttingen 1989

[5] G. Elter, M. Neuhaus, A. Meller, D. Schmidt-Bäse: J. Organomet. Chem. 381 (1990) 299

[6] M. Geschwentner: Staatsexamensarbeit, Universität Göttingen 1990

[7] S. Weber: Dissertation, Universität München 1984

[8] P. Paetzold, C. von Plotho, G. Schmid, R. Boese, B. Schrader, G. Goudeard, U. Pfeiffer, R. Gleiter, W. Schäfer: Chem. Ber. 117 (1984) 1089
[9] M. Haase, U. Klingebiel, R. Boese, M. Polk: Chem. Ber. 119 (1986) 1117

[10] G. Elter, O. Glemser, W. Herzog: J. Organomet. Chem. 36 (1972) 257

[11] G. Elter, A. Meller, W. Luthin: Z. anorg. allg. Chem. 560 (1988) 18

Korrespondenzanschrift:

Prof. Dr. A. Meller

Institut für Anorganische Chemie der Universität

Tammannstr. 4

W-3400 Göttingen, Bundesrepublik Deutschland 